Health Sociology

Review

Routledge

Taylor \& Francis Group

\title{
Purifying and hybridising categories in healthcare decision making: the clinic, the home and the multidisciplinary team meeting
}

\begin{tabular}{|c|c|}
\hline Journal: & Health Sociology Review \\
\hline Manuscript ID & Draft \\
\hline Manuscript Type: & Original Paper \\
\hline Keywords: & $\begin{array}{l}\text { Healthcare decision making, Categorisation analysis, Latour, Healthcare } \\
\text { settings }\end{array}$ \\
\hline Abstract: & $\begin{array}{l}\text { This article explores processes of legitimising of health knowledge in three } \\
\text { different spaces, the clinic, the home and the multidisciplinary meeting. It } \\
\text { considers the ways in which categorisation work, health understandings } \\
\text { and therapeutic actions are intertwined. The analysis draws on } \\
\text { ethnomethodology and actor-network theory to suggest that in the clinic } \\
\text { consultation room a number of interactional elements in talk about side } \\
\text { effects and talk about unorthodox interventions attempt to stabilise the } \\
\text { categories used by health experts. In contrast, the household is a centre of } \\
\text { knowledge production that may subvert, manipulate or align with expert } \\
\text { systems. Fixed and stable expert knowledge becomes flexible and } \\
\text { hybridised inside the home. In the multidisciplinary team meeting different } \\
\text { forms of authority that are called upon in hospital-based cancer care } \\
\text { meetings, where health professionals mix scientific understandings with } \\
\text { other ways of knowing in determining treatment options. From these } \\
\text { empirical observations it is argued that therapeutic actions are not subject } \\
\text { in any simplistic way to a dominant mode of understanding, but there are } \\
\text { many means of understanding that selectively come into play in relation to } \\
\text { the specifics of the interaction that is occurring between patients, health } \\
\text { professionals and therapeutic spaces. }\end{array}$ \\
\hline
\end{tabular}

\section{SCHOLARONE \\ Manuscripts}




\title{
Purifying and hybridising categories in healthcare decision making: the clinic, the
} home and the multidisciplinary team meeting.

\begin{abstract}
This article explores processes of legitimising of health knowledge in three different spaces, the clinic, the home and the multidisciplinary meeting. It considers the ways in which categorisation work, health understandings and therapeutic actions are intertwined. The analysis draws on ethnomethodology and actor-network theory to suggest that in the clinic consultation room a number of interactional elements in talk about side effects and talk about unorthodox interventions attempt to stabilise the categories used by health experts. In contrast, the household is a centre of knowledge production that may subvert, manipulate or align with expert systems. Fixed and stable expert knowledge becomes flexible and hybridised inside the home. In the multidisciplinary team meeting different forms of authority that are called upon in hospital-based cancer care meetings, where health professionals mix scientific understandings with other ways of knowing in determining treatment options. From these empirical observations it is argued that therapeutic actions are not subject in any simplistic way to a dominant mode of understanding, but there are many means of understanding that selectively come into play in relation to the specifics of the interaction that is occurring between patients, health professionals and therapeutic spaces.
\end{abstract}

Keywords: Healthcare decision making; Categorisation analysis; Latour; Healthcare settings 


\section{Introduction}

This article explores the legitimising of health knowledge in three different spaces, the clinic, the home and the multidisciplinary meeting, and considers the ways in which categorisation work, health understandings and therapeutic actions are intertwined. It illuminates the varieties of sense-making in these spaces and challenges assumptions about expertise and truth production. The three examples chosen allow reflection on the different kinds of categorisation work undertaken by householders, patients and practitioners and how categories of people, as bearers to therapeutic action, are stabilised and hybridised and how categories are used as resources to justify decision-making.

The data is drawn from different research programmes. Data from the clinic is taken primarily from an archive of health related interactional data. Data from households is taken from a research programme on the social meanings of medication involving 55 households with interview data, diaries and photos being the primary resources. Data from the multidisciplinary team is taken from research on cancer care, and there is also an account about a consultation taken from this data. References to this material is provided where the data is being discussed.

The theoretical perspective taken is broadly ethnomethodological in approach. In ethnomethodology the researcher seeks to describe the methods people use when they are "doing" social life (ten Have, 2004). A goal of this orientation is to identify the interactional work that talk does in the context in which we see it being done (Lepper, 2000).

Ethnomethodologists are not only concerned with what is said, but also with the action being performed in the interaction such as a request or a justification. Garfinkel, the founder of ethnomethodology, was interested in the procedures that people used to make sense of "the circumstances in which they find themselves" (Heritage, 1984, 4). One concept discussed below in relation to the data is the documentary method of interpretation, where people interpret events on the basis of their understanding of an underlying pattern (ten Have, 2004). Douglas provides a well-rehearsed example of this in his argument that suicide is an 'achievement' that results from the sense-making activities of coroners in terms of what they consider to be typical circumstances, motives and so (Douglas, 1970). Suicide rates reflect these understandings. The event of suicide is interpreted in relation to prior understandings about suicide, so in turn reinforcing those understandings.

Two approaches used to try to elucidate people's methods are the analysis of membership category devices and conversation analysis. Membership Categorisation Analysis (MCA) allows us to identify what identity categories respondents use (such as client/customer/patient) and the consequences of such categorisation (Stokoe, 2012). MCA has been described as the analysis of 'social structure in action' (Lepper, 2000). A membership categorisation device (MCD) is a collection of categories with rules on how these categories are applied. For example in the MCD of 'the clinic' there are particular categories such as clinician and patient, and with each of these categories there are category bound activities such as responsibilities and obligations. A category that is made relevant in any particular situation is tied to a characteristic or expectation of that category, called a predicate. For example a predicate of being a 'clinician' is to 'treat'. People can identify with different categories contemporaneously and what category we identify with or are identified as influences how we are expected to act and how we are perceived. But these categories should not be thought of as something that one simply learns through processes of socialisation and then applies in the relevant setting. Action express categories and what 
categories being expressed inform us of the activity being undertaken. The categories are occasioned by the activity (Watson, 2015).

Conversation analysis (CA), involves an analysis of the sequential unfolding of interactions to identify how and what issues are recognised and attended to by the participants. CA assumes that all utterances perform social actions and attends to these actions by asking why this utterance is in this place at this time (Heritage \& Maynard, 2006). For this reason, CA transcripts provide more detail than orthographic transcripts, including the identification of pauses, overlap in talk, and changes in intonation.

\section{Hybrids and the moderns}

In addition to ethnomethodology the argument in this paper is influenced by Latour. According to Latour, ethnomethodology and Actor-Network Theory share the resource of accountability in their methodological approach (Latour, 2005). For both Latour and Garfinkel sociology could be described as a "science accounting for how society is held together" (Latour, 2005, 13). This paper draws on Latour's concept of hybrids to analyse knowledge stabilisation and decision making. Latour argues that what he calls "the moderns are a type of people who maintain a belief in the existence of pure categories, such as the scientific, the economic, the political, the cultural, the local, the global" but at the same time we are surrounded by "unruly hybrids that churn up all of culture and all of nature on a daily basis" (Blok \& Jensen, 2011, 55). This paper explores the categories at play in three different therapeutic spaces and it notes ways in which purification and hybridising processes play out.

Heidegger states that despite differences in modern ways of revealing, "[m]odern science's way of representing pursues and entraps nature as a calculable coherence of forces" (Heidegger, 1977, 303). Who makes nature coherent and in what space illuminates or reveals what is appropriate, legitimate and credible, but we cannot assume what sort of coherences are arrived at or how they are arrived at. We need to empirically ground our interpretation of who determines coherence and how it is achieved, and in doing so our assumptions and expectations may be challenged. What is legitimate, or normal, or usual, or accessible, depends on particular relationships between spaces, people, knowledge, practices and things.

In the clinic processes of purification teases out what resource is appropriate to use - and this purification process is based on particular notions of science and rationality. This contrasts with inappropriate resources, such as the use of understandings that are different from orthodox science or that are based on intuition. As we shall see the attempt to order the world in this way can only partially succeed. In the home many activities are performed, including leisure activities, life-sustaining activities, reproducing the population activities. A home could then be conceptualised, drawing on Latour's hybrid notions, as a clinic-home, or a home-clinic, but it is also a theatre-home, a restaurant-home, a school-home and so on. In addition to a hybrid space and a space of hybrids homes, like clinics and hospitals, are nodes of therapeutic activity, linked to many other nodes (including clinics and hospitals, but also other homes, workplaces, healing centres and so on), or in more accessible parlance, a therapeutic centre in its own right. Many more therapeutic forms can be drawn on in the home than in the clinic, including those approaches advocated in the clinic. But householders are not under the same constraints of audit and disciplinary practices as clinicians. In the Multidisciplinary meeting the mix of accounts of bodies doing things to other bodies, images on screens and pathology reports can make the ordering quite variable. What is appropriate is not always clear, or consistent or standardised. The individual experience of the clinician can, 
in particular instances, be the salient form of decision-making authority, and may be as unruled and undisciplined as the household.

In sum, the orientation of actor-network theory and ethnomethodology are deployed to consider the way in which categories are deployed and hybridised and used as resources in different spaces in therapeutic decision-making.

\section{Maintaining therapeutic coherence in the clinic}

In the clinic purification of therapeutics is activated in the de-legitimisation of challenges to expert understandings and non-orthodox medical knowledge. It is not that hybridising does not occur, or that categories don't get messed up. Rather, certain forms of truth production are not well accommodated - such as ways of knowing based on the understandings of nonexpert individuals, or on intuition, or on understandings arising from other therapeutic domains.

This first example looks at how side effects are discussed, providing us with some understanding of why side-effects are routinely underreported (for a discussion of underreporting of adverse drug reactions see Lopez-Gonzalez et al., 2009). From consultation data an interactional 'block' to reporting of side effects can be identified, and hence our 'knowledge' of side effects is reinforced (See Anon1 for a fuller discussion of side effects talk in the consultation). We can draw on Garfinkel's documentary method of interpretation to help us understand this (ten Have, 2004).

When concerns are raised about drugs with well-known side effects GPs will attend to them carefully. Consequently these side effects may get reported to drug monitoring agencies. But GPs are likely to dismiss or downplay concerns about side effects from drugs without a high profile for having side effects - resulting in side effects not being reported (Anon 1). So the reporting occurs in relation to a belief in an underlying pattern of side effects, and reinforces or stabilises that pattern. In this side effects example we can see how health knowledge is 'stabilised' in the clinic.

In this extract, an illustrative example from a larger data set, the patient provides an extensive preamble where he mentions his rash and who he has talked to about it and then goes on

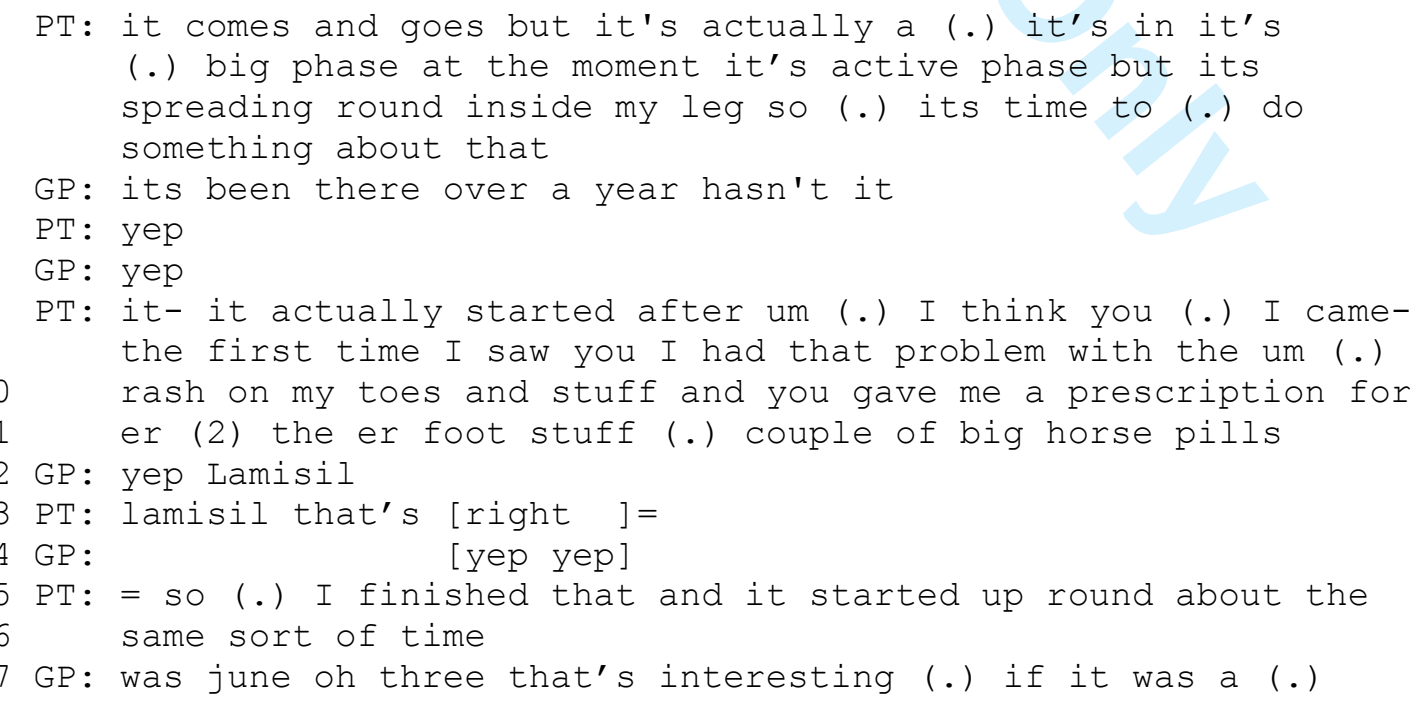


(For CA transcription conventions see

http://homepages.lboro.ac.uk/ ssah2/transcription/transcription.htm

The extract demonstrates the in situ enactment of clinical and patient categories, with their associated rights and obligations. The patient has very cautiously presented a claim that a medication prescribed by this clinician is the cause of his complaint. Features of the caution include the repairs on line 8 and 9 where the patient repeatedly starts to speak only to pause and re-start: "it actually started after um (.) I think you (.) I came- the first time I saw you". This suggests the patient is having some trouble finding the right way to present his case. The patient starts with "it actually started after" suggesting he might be going to say something like "after you prescribed that drug". This direct statement has the potential to unsettle the kind of category bound activities in this institutional space. Clinicians have the rights to determine causation of illness and disease and to prescribe medications. The patient attempts to respond to this interactional trouble by moving to "I think you". This too is troubling as the patient is still claiming the right to consider causation, so now he moves to "the first time I saw you", where any rights to diagnose are downplayed. The patient appears to move from what could be a direct proposal - it started up after you gave me the drug, and I think the drug was the cause - to a non-attributive tacit linkage proposal (Gill \& Maynard, 2006) that we get on lines 15 and 16: "I finished that and it started up round about the same sort of time". Through this interaction the patient has enacted the patient role and ceded the right to diagnose to the doctor. The clinician recognises what is being proposed here immediately and moves to provide reasons for rejecting the proposal, which is done with a delicacy so that the proposal is not positioned as an unreasonable one, for example seen in lines 18 and 19 where he says "you can get a reaction to any of them". The doctor enacts his role through the interaction in retaining the right to diagnose, prescribe and attend to the needs of the patient.

This is an illustration of a common feature in interactions around side effects (with some exceptions) where the default position for the clinician is to deny or minimise any association of a prescribed medication with signs and symptoms experienced by the patient (Anon1). An explanation for this is that clinicians deploy the documentary method of interpretation where understanding uses what we know and what we are told. The appearance points to (documents) a presupposed underlying pattern and the appearance is at the same time interpreted on the basis of the presupposed underlying pattern. This then reinforces the expert role and purified 'knowledge' in this setting.

In this first example we see the in situ enactment of the relevant institutional categories that stabilise the roles and activities of the clinic. In the next example we see something similar with the categories of doctor and patient stabilised, but in this instance the patient has demonstrated a clear awareness of a transgression that he makes when he suggests that he wants to follow an alternative diet. As opposed to the first extract, where the patient appears to be aware of the potential contentious nature of his claims, and so presents the claim in a heavily mitigate fashion, in this example the patient 'blunders' into a transgression. The participants rapidly determine that an idea is incoherent and not allowable. This example is from a part of a consultation where the clinician is looking at the computer and talking to the patient (this and the following example are taken from Anon2).

1 GP: and um (.) now you had blood tests (.) they were all last 
A feature to note occurs on lines 13 and 14. At this point the patient has explained what he wants the tests for, referencing an unorthodox approach to dieting. The clinician turns to the patient and before she has articulated her alternative perspective on this the patient has already made a retraction: "GP: oh yeah ((inhales)), PT: yeah (.) or not". From here on the patient strongly affiliates with anything the clinician says, seen in the array of "yep" and other alignment utterances in lines 17, 19, 22, 24, 26, 27, 29, 31, 33 and 35. We can see from this how attuned the patient is to 'illegitimate' knowledge, how quickly positions can change, how little evidence is required when one 'legitimate' view dominates, and how little effort is made to understand the reasons for holding 'illegitimate' views. The expert role, and the purified category of what could be termed orthodoxy (purportedly based on science and rationality) is interactionally enacted, but we can be cautious about what might happen outside of the clinic here. The patient has aligned interactionally, but not necessarily cognitively.

The third example looks at how a contested claim is more thoroughly worked through, and how in this case reasoning does occur, which challenges the enactment of the categories of doctor and patient. The patient does not enact a passive role, which interactionally leads to a 
compromise of sorts. In this case we can suggest, at least tentatively, that efforts to maintain purified categories of knowledge have not been successfully stabilised.

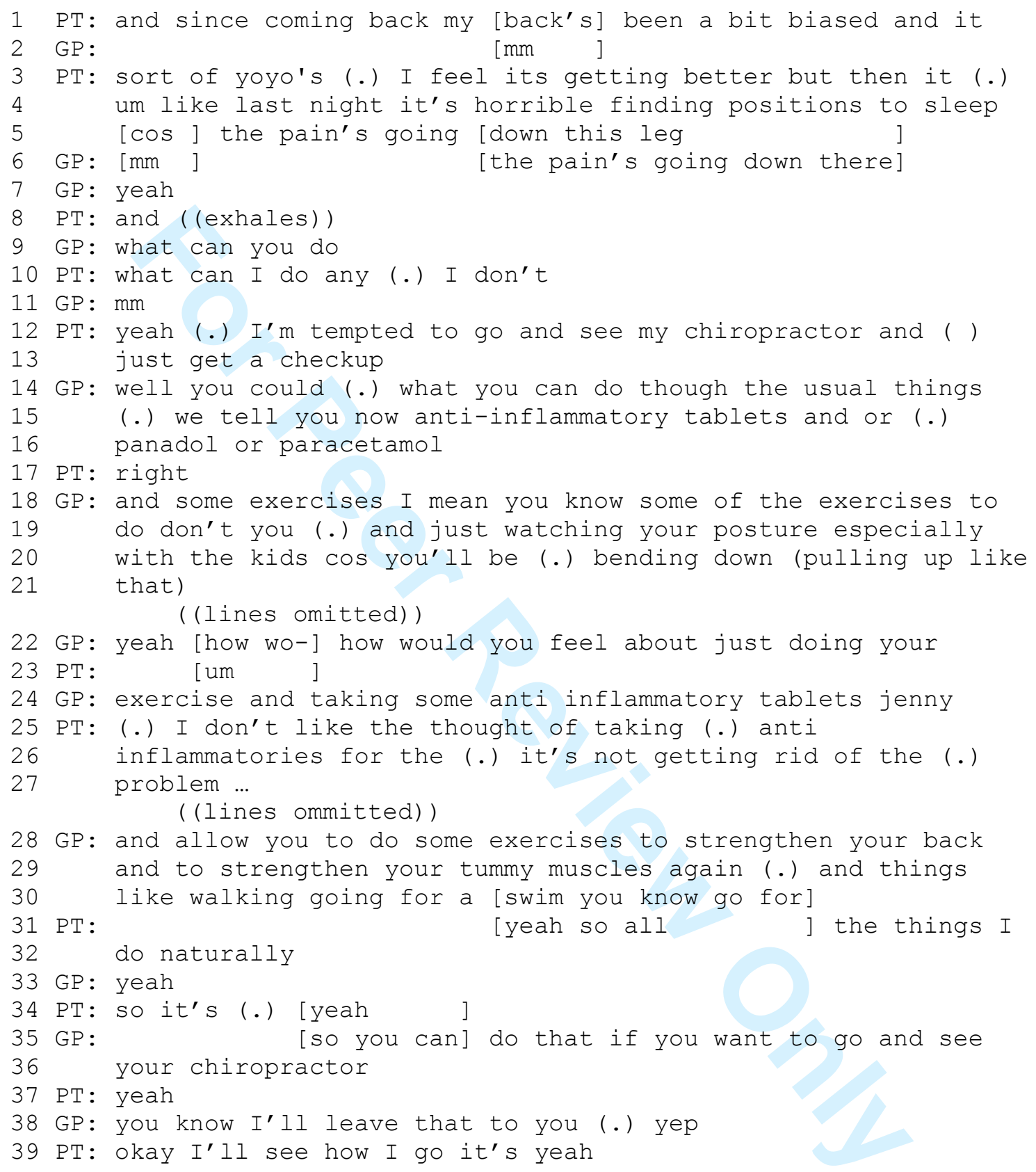

We can see here that the patient mitigates the proposal to see her chiropractor, an activity that would mess up the purified categories of the orthodox medical consultation as the chiropractor may hold alternative understandings of the problem that the patient presents with. The mitigation is seen in the patient stating that she is "tempted" (line 12), but it is clear that the activity of consulting a chiropractor is not unusual in that it is "my chiropractor". The GP attempts to dissuade the patient from this activity by offering a contrasting approach starting at line 14. The patient is overt in not aligning with this contrasting plan with "I don't like the thought of taking anti inflammatories" on line 25 and 26. She again resists the proposal being made by her GP on line 31 and 32 with "all the things I do naturally", so 
signalling that she already follows the suggestions being made but by implication they have not resolved her complaint. At the end of this script we see an alignment of sorts, with the GP saying you can "see the chiropractor", so allowing for the mixing of different therapeutic approaches, and the patient stating that she will "see how I go", and so suggesting the possibility that the mixing might not take place. All options are now available, but may or may not be pursued. The GP's attempt to work with 'purified' categories is reluctantly conceded in the face of persistent opposition from the patient. Here there is a failure to reinforce the expert role or purified categories.

The final example from the consultation space illustrates the maintenance of purified categories, but with likely negative consequences (see Anon 3). In this example the patient recalls an incident where a treatment plan in response to a cancer diagnosis is being made. The consultation is between a Māori patient and a non-Māori health practitioner, where the patient provides an account of her response to suggestions to have orthodox treatments for the cancer:

I said, "No, I'm not going that way. I'm going to stay with the rongoa". The Māori herbal way. Because it was a holistic approach. And it clashed straight away...I walked out of that meeting. I ran out...I basically said, "Get fucked to you," and left... Because I didn't like... the fact that he could sit there and pooh pooh...my way of wanting to get it fixed at that time. And basically telling me I'm a dead person if I go the rongoa way.

In this instance the categories of doctor and patient as a pairing is not enacted as the patient does not take on the patient role. However, a reinforcing of 'purified' categories occurs where biomedicine and rongoa medicine stay separate and unhybridised.

The examples above provide evidence of different ways in which categories or forms of knowledge can be purified and stabilised or mixed up and undermined. There are differences between patients and practitioners in relation to what therapeutic technologies and ways of knowing are allowable. There are different means of understanding that are being accessed in these various interactions. In the case where patients table something unorthodox - a diet, a consultation with a chiropractor and the use of indigenous healing approaches - the patient may well have greater access to these different means of understanding. Patients may have acquainted themselves with what is unorthodox, and may well have more knowledge of what is unorthodox than the GP. This may make it more difficult for the clinician to enact expertise. If they do so, the patient may withdraw from that space - as in the last case with the use of rongoa.

In contrast, in side effects talk the patient has only their understanding to call upon, and not some other means of understanding. The GP as the prescriber of the medication enacts the role of expert. The patient concedes when confronted with this expertise. Turning to the household space, the means to understanding can be considerably expanded, and it is much more difficult for a non-present practitioner to purify categories of therapeutic activity.

\section{Coherence of therapeutic forces in the home}

At the household level we witness how categories that might appear fixed and stable outside the home are rendered flexible and hybridised inside the home. There are diverse means of understanding that can be and are drawn upon. The household can be viewed as a therapeutic 
centre in its own right (Anon4). Unlike clinics, where information is gathered in a series of one-off visits, the household is a space where health and illness are constantly monitored, observations are continuous, experimentation is consistently undertaken and a vast array of resource material is assimilated and assessed.

In the home the householder can still enact a passive patient role ceding all authority to the expert consulted in other therapeutic spaces. Prescribed medicines can be viewed as the answer to health problems and as restoring order where order has been lost. Decision-making can be completely ceded to the health expert as the householder can just "do what the man says" as one householder states when confronting the bewildering range of medications he takes: "Cholesterol pills. Gout tablet. These are for my diabetes. This is something for blood pressure, I think. These ones look after my liver. I don't know what they do but I have some of them every day anyway" (Anon5). In such cases the householder does not have to have any means of understanding other than through the advice of a clinician. The coherence of therapeutic forces is maintained as other options do not need to be weighed up.

However, in many households the means of understanding are varied and vast. Whereas we can see activities in consulting rooms to purify health practices (science, orthodox, natural etc.), householders hybridise these categories. Homes engage in 'truth production' through research, experimentation and observation, but this form of truth production is not readily visible or readily disciplined. Paula stops taking medication for osteoporosis as she has linked this to stomach pains she is getting - but she does not tell her doctor about this. Ingrid knows when she is taking too much vitamin $\mathrm{C}$ because that is when she starts getting diarrhoea, and Louisa experiments with her homeopathic medications to work out how many drops she needs to relieve her of a urinary tract infection Anon 6). Different understandings are hybridised in making sense of therapeutic interventions, where the home becomes a homelaboratory for testing and titrating.

In the home we see the activities of diagnosing and prescribing. Lindsay Prior has argued that people are not skilled or practised in diagnosis (Prior, 2003), but research shows that diagnosis in the household is ubiquitous (Anon6). There is a need to 'diagnose' before seeking outside help. Diagnosis is based on close observation of what is normal. Mothers make assessments about how sick their children are by referencing prior bouts of illness. Householders make their own assessments about whether they need to seek outside help. Hazel does not take her child, who has a fever, to her doctor as she knows that "is the way that she gets sick" (Anon6) In the household, professional help comes second. On diagnosis, householders may prescribe folk remedies, supplements, changes of diet, exercise and routines.

When advice is sought from outside the household it is adapted and modified in terms of practical exigencies and particular understandings. Householders may deviate from recommendations by stopping medications, rejecting medications or ignoring advice and warnings. Dave takes alcohol along with his over-the-counter medication with its warning not to have it with alcohol because he is concluded that it is "not very dangerous".

Recommendations from CAM practitioners may also be adjusted. Cost of supplements for Paula are too high, so she does not take the recommended dose. Householders may mix orthodox medications with alternative ones against their alternative therapist's expectations, with Ingrid suggesting that her Ayurvedic practitioner would "have a heart attack" if he found out (Anon6). The Ayurvedic practitioner can then continue to believe that his purified practices are followed, whereas his patients happily hybridize away. 
Householders could also have their own expertise in certain approaches or areas.

Householders may have specific training in therapeutic approaches, such as aromatherapy, or have family-based knowledge, such as in the use of homeopathy. Householders can become experts in their children's or their own health. Householders may engage in experimentation to determine such things as the right dose for themselves or dependents, when to take medications, when to avoid medications and to find what works. There is a huge variety in the sources of practices, spanning across generations, workplaces, friends, relatives, health advisors, support groups, individual research (Internet, libraries) and also opportunistic advice (popular media).

To conclude this section, householders deconstruct the scientific 'facts' and theoretical purity, they have their own hierarchies of sources, are active producers of hybridised medication practices and undertake work and responsibility in relation to therapeutic practices. Medicine was never contained to the locations of experts in consulting rooms and hospitals, but outside are spaces that are unruled and undisciplined.

\section{The multidisciplinary meeting - justificatory work of categories}

In the multidisciplinary meeting (MDM) decisions, such as treatment plans, are made when health professionals come together to discuss patients. A range of means of understanding are drawn upon that can be related to sources of authority. Particular forms of categorisation work can also be undertaken when actions need to be justified. In this space health professionals draw on a range of persuasive devices to achieve credibility when they do not pursue what might be seen as a 'recommended' or expected course of action (Anon7).

The means of understanding in MDMs are quite varied. Two that we might expect given that these meetings take place in a hospital setting are a means based on a technological authority, from diagnostic and pathological evidence presented, and a research authority, from previous findings. These are the expected purified forms. But participants in MDMs may also draw on their own clinical experience based on their own encounters with the type of condition being discussed. Authority can also come from referral notes, particularly when participants in the meeting have not met the patient being discussed, as occurs when referrals are received from other centres.

Other forms of authority to make a decision are in more hybridised forms, mixing clinical knowledge with categorisation work. One means of understanding is through 'encountered' authority, which is the authority given to someone who has met the patient. If no team member has encountered the patient discussions can be difficult as all information may not be at hand. The person who has met the patient has indisputable knowledge. An elderly patient being discussed is assessed as high risk for surgery, but the presenting surgeon has met the patient and determined that he "works reasonably well" - so surgery is the outcome (Anon7). The encounter with the patient is drawn on to claim the right to categorize the patient in a particular way, and so justify an action that the 'pure' form of research based decision making would not support. The expected purification of therapeutic decisions in the hospital settings is de-legitimised by a subjective claim to knowledge. Although participants to the MDM are health professionals, the particular rights of health professionals are enacted in contingent ways. 
It is through encountered authority that categorisation work that enacts cultural understandings come into play. To take another example, occupation is very rarely mentioned so when it is we can consider the work that the deployment of this category does. For instance, where a presenting surgeon notes that the patient is a colour therapist, a cultural knowledge about colour therapy means there is no need to elaborate on "it is my guess that she will decline surgery" (Anon7). The category of colour therapist is associated with activities such as adhering to unorthodox understandings of healing and not taking the advice of biomedical experts who do not accept the therapeutic value of colour therapy, and so the colour therapist may be expected not to follow the surgeon's advice. Rather than being constructed as a complex individual the purified category of 'unorthodox' is deployed in distinction from the purified category of orthodox surgery. The means to understanding here is a cultural one enacted through categorisation work, but not a technological or researchbased means of understanding.

Presenting surgeons can also draw on the authority of their own personal sense of touch. The "horrible tissue" that a surgeon encounters during surgery, understood by the surgeon in terms of "poor vascularisation", justifies a conservative approach. The technological apparatus show that there are vessels passing through the tissue, apparently contradicting the surgeon's claims, but the experiential authority of the surgeon is not challenged (Anon7).

Other categories used include prickly individuals, and frail individuals. These assessments justify particular actions taken. In these meetings technological presentations are the basis for discussion, and the science is generally assumed. But health professionals hybridise ways of knowing, particularly when their actions could be contested. Category work, of tissues and people in these cases, is a resource to justify actions, and science and individual understandings and experience can be hybridised and so messing up the purity of biomedical practice.

\section{Conclusion}

The GP clinic is a space of intermingling, with legitimised ways of knowing being enacted and also undermined. In the clinic patients and practitioners can be finely attuned to the categories being used, seen in the quick recognition of the categories at play and what are legitimate or illegitimate category bound activities. GPs may attempt to apply a biomedical category of healing, when patients bring their hybridising approach into the purified atmosphere of the clinic. GPs, like anyone else, use the documentary method of interpretation as outlined by Garfinkel in decisions about responding to patient concerns about side effects. In effect we can consider here that drugs are categorised - with some drugs 'known' to be linked to side effects, and others not having that predicate. So in both the CAM consult and the side effects consult GPs work to maintain categories that align with their work, whilst patients may artfully disrupt those categories. In the GP consultation room attempts are made to tame the unruly hybridised nature of everyday life.

In the clinic category work stabilises, successfully in the case of side effects talk but not so successfully in relation to CAM-talk. An ethnomethodological sensibility provides useful insights and unsettles assumptions. In homes and MDMs categories are subverted and hybridised, in the home as a response to pragmatic concerns and in the MDMs as a justificatory resource. Rather than the home being a passive therapeutic space 'science' like therapeutic activities take place. At the household level we witness how categories that might 
appear fixed and stable outside the home are rendered flexible and hybridised inside the home.

The MDM is heavily 'scientised', but also personal, subjective, and non-transparent ways of knowing can trump the science. In the MDM the power of the individual practitioner to lay legitimate claim to the importance of their direct experience can triumph, and categorisation work of patients can be used to justify or defend actions. Health professionals can undertake hybridisation work, where science is not applied but is mixed with other ways of knowing in determining treatment options and interactions with patients.

Latour's sociology of associations provides a means of analysing the stabilising of facts with a fact being a stabilised statement (Latour \& Woolgar, 1979). This provides a picture of scientific facts being stabilised and once so stabilised the work of re-negotiation becomes very difficult. It is difficult to "cast into doubt" the facts and technologies when the processes of their negotiation have become opaque (Lindemann, 2011). In this way scientific facts can "confront actors like an exterior force" (Lindemann, 2011, 101). In contrast, ethnomethodology, according to Garfinkel, focuses on in situ practices of actors "taking into account the indexicality of meaning in particular expressions in a particular context of embodied action" (cited in Lindemann, 2011). Garfinkel turns our attention to the everyday, and we can see how efforts to stabilise can shape interaction (as in side effects talk in the consultation), but also how in the everyday and in the process of interaction the apparatus of science and fact building can be destabilised. In the everyday the encounter, the direct experience, can be the basis for therapeutic action. It is not so hard to open the black box of fact stabilisation in everyday experience, as it may be to open the black box through formal institutions such as the scientific journal and so on.

Whereas Latour emphasises durability Garfinkel emphasises contingency. These tendencies can be seen in a dialectical relationship, where the durable systems embedded in centres of calculation are contingently drawn on in practice, and it is that contingency that has been considered here. By exploring the contingency we can get a better sense of health care decision-making, and avoid making assumptions about the passive response of actors to the reach and oversight of centres of calculation. The 'stabilised' facts are always up for negotiation in terms of how and if they will have their effect in situated practices.

Latour does provide us with some conceptual tools to think through what happens in these situated practices. His use of the concept of hybrids is one such tool. But the attention to the social practice that ethnomethodology directs us to, and the focus on the sense making activities of actors, provides insights into the processes of hybridisation and purification. Therapeutic actions are not subject in any simplistic way to a dominant mode of understanding, but there are many means of understanding that selectively come into play in relation to specifics of the interaction that is occurring between patients, health professionals and the spaces they inhabit.

\section{References}

Anon1

Anon2

Anon3

Anon4

Anon5 
Anon6

Anon7

Blok, A., \& Jensen, T. (2011). Bruno Latour: Hyrbid thoughts in a hybrid world. London and New York: Routledge.

Douglas, J.D. (1970). Understanding everday life. In J.D. Douglas (Ed.), Understanding everyday life: toward the reconstruction of sociological knowledge pp. 3-44). Chicago: Aldine.

Gill, V., \& Maynard, D.W. (2006). Explaining illness: patients' proposals and physician' responses. In J. Heritage, \& D.W. Maynard (Eds.), Communication in medical care: interaction between primary care physicians and patients pp. 115-150). Cambridge: Cambridge University Press.

Heidegger, M. (1977). The question concerning technology. In D.F. Krell (Ed.), Martin Heidegger: Basic Writing from Being and Time (1927) to The Task of Thinking (1964) pp. 287-317). New York: Harper \& Row.

Heritage, J. (1984). Garfinkel and ethnomethodology. Cambridge: Polity Press.

Heritage, J., \& Maynard, D.W. (2006). Introduction: analyzing interactions between doctors and patients in primary care encounters. In J. Heritage, \& D.W. Maynard (Eds.), Communication in medical care: interaction between primary care physicians and patients pp. 1-21). Cambridge: Cambridge University Press.

Latour, B. (2005). Reassembling the Social: An Introduction to Actor-Network-Theory. New York: Oxford.

Latour, B., \& Woolgar, S. (1979). Laboratory life: the construction of scientific facts. Beverly Hills: Sage.

Lepper, G. (2000). Categories in text and talk: A practical introduction to categorization analysis. London: Sage.

Lindemann, G. (2011). On Latour's social theory and theory of society, and his contribution to saving the world. Human Studies, 34, 93-110.

Lopez-Gonzalez, E., Herdeiro, M.T., \& Figueiras, A. (2009). Determinants of underReporting of adverse drug reactions: A systematic review. Drug Safety, 32, 19-31.

Prior, L. (2003). Belief, knowledge and expertise: the emergence of the lay expert in medical sociology. Sociology of Health \& Illness, 25, 41-57.

Stokoe, E. (2012). Moving forward with membership categorization analysis: Methods for systematic analysis. Discourse Studies, 14, 277-303.

ten Have, P. (2004). Understanding qualitative research and ethnomethodology. London: Sage.

Watson, R. (2015). De-reifying categories. In R. Fitzgerald, \& W. Housley (Eds.), Advances in membership categorisation analysis pp. 23-49). London: Sage. 